\title{
SYNTHETIC PUBOVAGINAL SLING (TVT): FAILURE IN CONSERVATIVE TREATMENT FOLLOWING VAGINAL EXTERIORIZATION
}

\author{
EDGAR THORELL, GIOVANI T. PIONER, MARIANA R. THORELL
}

\author{
Santa Casa de Porto Alegre, Porto Alegre, Rio Grande do Sul, Brazil
}

\begin{abstract}
Female, 57 year-old patient, reported having undergone surgery for correction of urinary incontinence due to sphincteric insufficiency with the implantation of a synthetic pubovaginal sling 14 months earlier. Though she did not present urine loss any longer, approximately 60 days following the surgical procedure she started to report dysuria, pollakiuria and dyspareunia. Attempts of a conservative solution were ineffective. The appearance of a vaginal infra-urethral granuloma and the exteriorization of the synthetic material led to its removal.
\end{abstract}

Key words: urinary incontinence; urinary sphincter; prostheses and implants

Int Braz J Urol. 2004; 30: 123-124

\section{INTRODUCTION}

We describe a case of vaginal exteriorization following the use of the Prolene polypropylene tape (TVT device from Ethicon Johnson \& Johnson), which required its removal 14 months after its insertion.

\section{CASE REPORT}

Female, 57-year old patient complained of dysuria, pollakiuria, urinary infection and dyspareunia, which produced intense discomfort, with worsening of symptoms in the last 40 days. She reported that 14 months earlier she had undergone an implantation of a synthetic pubovaginal sling in another hospital for correction of urinary incontinence, due to intrinsic sphincteric insufficiency. She had presented prolonged urinary retention during the post-operative period, which forced her to stay in hospital for 5 days. During the first 60 days the manifestations of urinary incontinence had ceased, but a picture of pollakiuria, dysuria and dyspareunia progressively emerged. One year after surgery she noticed the formation of a vaginal nodule and the sensation of a solid material on digital contact at the infra-urethral region, which prevented sexual intercourse. She was medicated and oriented to maintain the follow-up. When she came to our service, she presented urinary infection and, on the vaginal inspection, it was possible to observe the presence of a granuloma and minimal exteriorization of the synthetic material through the mucosa. The urethrocystoscopy under sedation showed integrity of the urethral mucosa and the specular examination confirmed a mild exteriorization $\mathrm{f}$ the synthetic tape through the vaginal mucosa, not exceeding $0.5 \mathrm{~cm}$ of exposed area. The device's removal was suggested and accepted by the patient, since conservative treatment had already been tried and the patient complained of intense discomfort. Tape removal was performed similarly to its insertion, with a small incision in the vagina anterior wall and, in both sides, a careful peri-urethral dissection. The tape was adhered to adjacent tissues, as well as covered by fibrous tissue, differently from the mesh aspect present by the material before use. A 5-cm long segment was re- 
moved, which encompassed the urethra. The adherence to tissues made a larger dissection difficult. There was immediate improvement in the complaints, with the patient remaining continent for approximately 6 months. Due to the reappearance of urinary incontinence, the patient underwent a new evaluation and, the urodynamic study reveal a bladder with normal capacity, complacence and proprioceptive sensitivity, absence of vesical instability or infravesical obstruction. A new surgical repair was proposed, performing the technique of autologous aponeurotic sling made of rectus muscle of abdomen, without identifying any sign of the presence of TVT, despite only one 5 to 6 -centimeter segment had been removed. After more than 10 month since this last procedure, the patient is perfectly continent.

\section{COMMENTS}

Palma et al. were the pioneers in our setting on the use of Prolene tapes as sling for surgical repair of incontinence (1). There are advantages e disadvantages concerning its use when compared to the technique of confectioning the "sling" from fascia lata or aponeurosis of rectus muscle of abdomen. Advantages are related to early return to activity, decreased use of analgesics, lesser surgical aggression, shorter surgical time, reduced stay in hospital, and it can potentially be performed at outpatient settings under local anesthesia local (2). Disadvantages are related to cost, which is considered high in our economy, limiting its use to a minority, in addition to the possibility of sling infection with risk of exteriorization, with such possibility being reported in the literature from $3 \%$ to $23 \%$, an event that hardly occurs with the use of autologous material.

Sweat et al. described both vaginal erosion and obstruction of the bladder neck produced by the polypropylene tape, suggesting also its complete removal if erosion occurs (2). Even though this approach appears to be a consensus among urologists, Kobashi $\&$ Govier recently described 4 cases of vaginal erosion following the use of polypropylene tape ( 3 cases with SPARC and 1 with TVT) where conservative treatment was successful, with occurrence of reepithelization (3). The same author suggests that if reepithelization does not occur within 3 months, its removal is mandatory and that one of his cases, even with an exposure area larger than 1 centimeter, presented a favorable outcome 6 weeks later (3). It is speculated that the urethral erosion can be due to excessive tension of the sling over the urethra, or exaggerated dissection impairing its thickness, and making it more susceptible to damage. In relation to vaginal erosion, it would be due to a subclinical infection of the synthetic material, producing an accumulation of secretions that would lead to exteriorization. There are reports in the literature on the persistence of urinary continence even after "sling" removal following erosion. Such situation did no occur in our case, since incontinence returned after less than 6 months, leading to a new surgical procedure.

\section{REFERENCES}

1. Palma PCR, Ikari O, D'Ancona CAL, Netto Jr.: Synthetic pubovaginal sling for treatment of stress urinary incontinence. J Bras Urol. 1992; 18: 202-4. [in Portuguese]

2. Sweat SD, Itano ID, Clemens JQ, Bushman W, Gruenenfelder J, McGuire EJ, et al.: Polypropylene mesh tape for stress urinary incontinence: Complications of urethral erosion and outlet obstruction. J Urol. 2002; 168: 144-6.

3. Kobashi KC, Govier FE: Management of vaginal erosion of polypropylene mesh slings. J Urol. 2003; 169: 2242-3.

Received: May 16, 2003

Accepted after revision: January 16, 2004

\author{
$\overline{\text { Correspondence address: }}$ \\ Dr. Edgar Thorell \\ Rua Tomaz Gonzaga, 430 / casa 13 \\ Porto Alegre, RS, 91340-480, Brazil \\ E-mail: thorell@terra.com.br
}

